\title{
Die Figuralmusik in Hrabyně um die Wende des 19. Jahrhunderts - Hrabiner Inventare
}

\section{Performing Practice of Figural Music in the Church in Hrabyně on the Beginning of the 19th Century - Hrabyně's Inventories}

Helena Kramářová / helena.kramarova@gmail.com

Ústav hudební vědy FF MU, Brno, CZ

\begin{abstract}
The study contributes on music in pilgrimage Church of the Assumption of the Virgin Mary in Hrabyne turn of the 18th and 19th century. The paper focuses on sheet of music inventories and church musical instruments (1804, 1819 and 1832). It reflects the purpose of genesis the inventories and included information about music in church, too. Important part identifies composers from inventory in 1819. We concentrate on church's organ and their repair, too. The work contains information about school in Hrabyně and teachers, who were teaching there and composing for the church. Nevertheless, Hrabyněs inventories are compared with other locations in Silesia and Moravia and the study also marginally handles conditions of performing practice of figural music in village, which are illustrated on schoolmaster Schenk's memories.
\end{abstract}

\section{Key words}

Church music, village school, figural music, composer of the $18^{\text {th }}$ century, turn of $18^{\text {th }}$ and $19^{\text {th }}$ century, Silesia, Moravia, Hrabyně 
Die Inventare wurden im kirchlichen Milieu seit dem späteren Mittelalter zu verschiedenen Gelegenheiten verfasst. Ihre Bedeutung hängt eng mit dem Alter und der Ausführlichkeit zusammen, deswegen sind vor allem die älteren Inventare in den wissenschaftlichen Kreisen von Interesse. Nichtsdestoweniger können auch die jüngeren über wertvolle Sachverhalte verfügen, die uns von anderen Quellen nicht bekannt sind, oder im Falle, wenn es an den primären Quellen mangelt. Die in manchen Hinsichten nicht so bedeutenden Inventare aus dem 19. Jahrhundert vermitteln dabei wichtige Informationen nicht nur über Zeit ihrer Entstehung, sondern auch über Geschichte der bestimmten Kirchen. Deshalb wird in dieser kurzen Abhandlung über das Musikgeschehen in der schlesischen Wallfahrts- und Dorfpfarrkirche in Hrabyně (Hrabin) durch das Prisma der Inventare berichtet.

Am Anfang muss betont werden, dass Hrabyně mehrmals durch Feuer und später im Krieg zerstört wurde und deshalb sind nur wenige Quellen in den Archiven erhalten, mindestens auf der tschechischen Seite. Allerdings befindet sich in Wien eine Menge an Archivalien, die nicht nur über Kirche, Schule oder Musik berichten, sondern auch viele Akten von den Wundertaten, die dank Mutter Gottes und ihrer Hrabiner Bildnis getan wurden. Diese Dokumente ungefähr aus der zweiten Hälfte 18. Jahrhunderts und dem 19. Jahrhundert sind im Zentralarchiv ${ }^{1}$ des Deutschordens bewahrt, weil Hrabiner Herrschaftsgut dem Deutschen Orden seit dem Jahr 1837 gehörte. In der Zeitspanne, die für diese Studie wichtig ist, war Hrabyně das Eigentum von der Adelsfamilie Mittrowsky. Zuerst ging Hrabyně von Anton Graf von Mitrovsky im 1832 an Ernst Otto Ritter von Badenfeld und anschließend an den Deutschen Ritterorden über. ${ }^{2}$ In diesem Aktenmaterial wird die Aufmerksamkeit hauptsächlich auf die Inventare aus den Jahren 1804, 1818 und 1832 gerichtet, sowie auf die Notizen, die das Gesamtbild des Musiklebens vor allem am Ende des 18. Jahrhunderts ergänzen. Beiseite gelassen werden die Visitationsprotokolle aus der Schule und Fundationen, Einkommen des Kantors sowie zahlreiche Beschwerden über Kantoren, natürlich nur, wenn es mit den Musikalien- und Musikinstrumenten bestand nicht direkt zusammenhängt. ${ }^{3}$

Die Berühmtheit der Hrabiner Wallfahrtskirche Mariä Himmelfahrt in Schlesien, genauer gesagt in Österreichisch-Schlesien stieg erst in der Hälfte des 18. Jahrhunderts. ${ }^{4}$ Hrabyně als Marianisches Zentrum wurde nicht nur von den Pilgern aus der Monarchie, sondern auch aus dem preußischen Teil des Schlesiens häufig besucht, die die Mehrheit bilden sollten. Eichler gibt an, dass die Pilger aus Mähren, aus Frýdek (Friedeck) und Místek (Mistek), aus Př́íbor (Freiberg in Mähren), aus Kelč (Keltsch), aber auch aus

1 Deutschordens Zentralarchiv (weiter nur DOZA WIEN), Fond Mei (Meisterthum) Inv. Nr. 432/1, Inv. Nr. 432/2, Inv. Nr. 433/1.

2 Vgl. HOSÁK, Ladislav. Historický mistopis země Moravskoslezské. VIII. Opavský kraj. Praha: Společnost přátel starožitností československých, 1937, S. 750.

3 Mehr zu diesem Thema in KRAMÁŘOVÁ, Helena. Chrámové inventáre hudebnin z přelomu 18. a 19. století. Hrabyňské inventáře [online]. Brno, 2013 [cit. 2016-09-28]. Bakalářská práce. Masarykova univerzita, Filozofická fakulta. Vedoucí práce Vladimír Maňas Zugänglich unter: 〈http://is.muni.cz/th/383033/ff_b/>.

4 Vgl. MALURA, Jan. Hora Olivetská Matěje Tannera a kultura poutních míst v baroku. In TANNER, Matěj. Hora olivetská. Brno: Host, 2001, S. 9. 
Moravská Ostrava (Mährische Ostrau) gekommen seien. ${ }^{5}$ Diesem Aufstieg half die neu gebaute, bzw. gemauerte Kirche, die der Freiherr Mathias Ernst und sein Sohn Ernst Benjamin von Mittrowsky in den Jahren 1723-1731 bauen ließen. ${ }^{6}$ Bei der Kirche begann dann ihre Tätigkeit die Bruderschaft des hl. Johann von Nepomuk im Jahre $1736,{ }^{7}$ die unter anderem mit dem Musikbetrieb zusammenhing.

\section{Hrabiner Inventare}

Bevor die einzelnen Musikinventare nähergebracht werden, muss die Frage gestellt werden, ob sie die ersten Belege der musikalischen Praxis in der Wallfahrtskirche sind, oder ob die Figuralmusik in Hrabyně früher zu datieren ist. Die ersten Belege der Figuralmusik in Dorfkirchen sind allgemein in der zweiten Hälfte des 18. Jahrhunderts zuzuordnen. In Hrabyně verlief es genauso. Der allererste Beleg der Figuralmusik geht aus der Festlegung einer Fundation am 29. Dezember $1761^{8}$ hervor - es sollte ein Schullehrer und zwei Vokalisten bezahlt werden, die Gottesdienste sowohl mit der Choral- als auch mit der Instrumentalmusik begleiten sollten. ${ }^{9}$ Die Anordnung einer Fundation muss aber nicht dringend die tatsächliche Praxis belegen. Anscheind wurde in Hrabyně die Figuralmusik schon zu dieser Zeit gespielt. Dies wird von einer Bemerkung im Inventar aus dem Jahre 1804 beweist - als eine der Eintragungen, die den Pfarrarchiv beschreiben, steht ein Faszikel mit dem Namen „Über die Veranstaltung einer Figuralmusik an höheren Tägen - Hrabin am 24ten Novemb 777."10

Die meisten Informationen über Figuralmusik stammen aus vier erhaltenen Inventare. Der älteste ist aus dem Jahr 1804 und wurde Dreÿtheiliges Inventarium ${ }^{11}$ genannt

5 Vgl. EICHLER, Karel. Poutni mista a milostivé obrazy na Moravě a v rakouském Slezsku. Brno: Dědictví sv. Cyrilla a Methoděje. Díl první, část druhá, 1888, S. 139.

6 Die Barockkirche wurde anstatt der alten ursprünglichen hölzernen Kirche, die im Jahre 1497 konsekriert wurde, gebaut. Das Aussehen der heutigen Kirche stammt aus den Jahren 1885-86. Vgl. SAMEK, Bohumil. Umělecké památky Moravy a Slezska. 1., A-I. Praha: Academia, 1994, S. 544.

7 In Hrabyně fungierte im Jahre 1736 gegründete Bruderschaft des hl. Johann von Nepomuk. Vgl. ACO 182 (Hlučín 1771): 1736. Überliefert ist ein Lizitationsprotokoll über Verkauf des Bruderschaftsvermögens aus 1784 in Mährischen Ostrau. Vgl. DOZA Wien, Mei 432/1. Die Bruderschaftsmatrikel wurde vom Stadtpfarrer in Ostrau Balcárek gekauft - dank seines Interesses ist bis heute der vergoldete, Beschlag der Bruderschaftsmatrikel erhalten, als Umschlag für Missal aus dem Jahre 1927. Für ausführlichere Informationen: MAŇAS, Vladimír - ORLITA, Zdeněk - POTU゚ČKOVÁ, Martina. Zbožných duši úl. Náboženská bratrstva v kultuře raněnovověké Moravy. Olomouc: Muzeum umění Olomouc, 2010, S. 97. und MAŇAS, Vladimír. Náboženská bratrstva olomoucké (arci)diecéze do josefínských reforem [online]. Brno, 2003 [cit. 2016-09-30]. Diplomová práce. Masarykova univerzita, Filozofická fakulta. Vedoucí práce Bronislav Chocholáč Dostupné z: <http://is.muni. cz/th/13678/ff_m/>, S. 112.

8 Im gleichen Jahr wurden die Altare - Hauptaltar und zwei Nebenaltare konsekriert. Die Altarbilder für Nebenaltar wurden von Troppauer Maler Günther im Jahre 1 gemalt. Vgl. DOZA WIEN, Fond Mei, Inv. Nr. 432/1. Inventar 1804.

9 DOZA WIEN, Fond Mei, Inv. Nr. 432/1.

10 DOZA WIEN, Fond Mei, Inv. Nr. 432/1.

11 DOZA WIEN, Fond Mei, Inv. Nr. 432/1. 
(Inventar 1804). Einem Inventar ähnliche Quelle stellt ein Brief ${ }^{12}$ dar, der vom Schullehrer als eine Art der Beschwerde im Jahre 1818 verfasst wurde (Brief 1818). Ein Jahr später ist ein Kirchen-Musicalien Inventarium ${ }^{13}$ (Inventar 1819) vom gleichen Verfasser entstanden, dass, wie schon aus der Überschrift hervorgeht, die Instrumente nicht reflektiert wurden. In dem Musikalien-Inventar sind die Gattungen angegeben, weiterhin die Komponisten und Tonart des Stückes. Letztes Inventar wurde im Jahre 1832 geschrieben und wurde Über die Instrumente und Musicalien bey Hrabiner Kirche ${ }^{14}$ genannt (Inventar 1832).

\section{Inventar 1804}

Als Reaktion an die Dekrete Nr. 224 und Nr. 753 der Erzbischöflichen Konsistorium in Olmütz wurde am 17. November 1804 das sog. Drë̈teiliges Inventrium ${ }^{15}$ (Inventar 1804) fertiggeschrieben und vom hiesigen Lokalkaplan unterschrieben. Auf dem Titelblatt ist der Zweck eingetragen, zu dem das Inventar entstanden ist: Das Inventar ist „Zu beybehaltung der gutten Ordnung, und nothwendigen Nachricht der Nachkommenschaft im Jahre 1804 verfasset worden. ${ }^{" 16}$ Wie aus seinem ursprünglichen Titel hervorgeht, wurde es in drei Teile gegliedert: Im Betref der Kirche, Im Betref des Hrabiner Local Beneficium und Im Betref des Wohngebä̈̈des des Beneficianten. Der erste Teil beinhaltet übliche Beschreibungen der Kirchenausstattung. Interessant sind hier ausführliche Einträge der Sachen, die für Prozessionen bestimmt wurden. Danach kommt die in zwei Spalten geteilte Beschreibung: „Ob dem Chor ist befindlich“. Die erste Spalte zählt die Musikinstrumente inkl. Gesangbücher auf, die andere dann die Musikalien, die leider nur nach der Gattung und Gesamtanzahl angeben.

\section{Brief 1818}

Zu den Inventaren kann auch einen Brief zugeordnet werden, obwohl er als kein solches gedacht wurde. Es handelt sich jedoch um einen Brief, der ähnlich wie die Inventare die Chorausstattung sehr genau dokumentiert, und zwar nicht nur die Anzahl der Musikinstrumente und Musikalien, sondern auch den Zustand. Er wurde am 22. September 1818 vom Schullehrer Leopold Franz Czurda ${ }^{17}$ als eine Art der Beschwerde verfasst, die zur Verbesserung den Aufführungsbedingungen führen sollte.

12 DOZA WIEN, Fond Mei, Inv. Nr. 432/2.

13 DOZA WIEN, Fond Mei, Inv. Nr. 432/2.

14 DOZA WIEN, Fond Mei, Inv. Nr. 432/1.

15 Diplomatische Transkription des Inventars - Anhang Nr. 1.

16 Ibidem., Inv. Nr. 432/1.

17 Trojan führt nur seinen Vorgänger Jakub Opavský an und bemerkt, dass im Jahre 1777 die Schule brannte. Vgl. TROJAN, Jan. Kantoři na Moravě a ve Slezsku v 17.-19. století: jejich sociální postavení, společenská funkce a význam ve vývoji národni hudebni kultury. Brno: Muzejní a vlastivědná společnost, 2000. 
Am Anfang weist Czurda auf die Tatsache hin, dass er weder offiziell als Cantor und Regenschori genannt werde, noch, dass es ihm die Kirchenmusikinstrumente und Kirchenmusikalien nicht übergeben werden. Überdies bemerkt er, dass es zwar all diese Gegenstände auf dem Kirchenchor gebe, aber sie fast unbrauchbar seien. Vor allem seien sie nicht zur Aufführung der anständigen Kirchenmusik genügend. Schließlich erwähnt Czurda, dass der Zustand so schlecht sei, dass er sogar seine eigenen Instrumenten für den Musikbetrieb nutzen müsse, wofür er wahrscheinlich nicht entschädigt werde. Aus diesen Gründen verlangte er Behebung dieses Zustandes mit einer kurzen Notiz, dass dessen Unterlassung zu einer Kollision führen könnte.

\section{Inventar 1819}

Wie aus dem vorherigen Brief hervorgeht, war die Situation auf dem Kirchenchor in Hrabyně nicht gerade optimal. Das soll auch ein weiteres Dokument ${ }^{18}$ bezeugen, das ein Jahr später vom gleichen Schullehrer geschrieben wurde. So ist ein KirchenmusikalienInventar am 29. Juli 1819 dem Direktorialamt geschickt worden, dass um einen knappen Brief, bzw. eine Zuschrift ergänzt wurde. Czurda bittet relativ eindringlich, dass zu einer ordentlichen Übergabe der Kircheninstrumente kommen soll. Der eigentliche Wert des Inventar 1819 stellt die Auflistung der einzelnen Musikalien dar. Es könnte als ein Beleg für den Einkauf den neuen Musikalien dienen. Die Musikalien wurden nach Gattungen sortiert, weiterhin wurde die Tonart angegeben und letztendlich der Komponist, falls er bekannt wurde. ${ }^{19}$ Die Musikalien vertreten die Musik für den ganzen Betrieb innerhalb des liturgischen Jahres und erfassen mehr als 90 Musikalien - sehr bedauerlich ist dabei, dass es in diesem Fall um kein thematisches Inventar geht.

\section{Inventar 1832}

Am 20. Juli 1832 wurde das letzte bekannte Inventar über Musikinstrumente und Musikalien in der Hrabiner Kirche geschrieben - verfasst wurde es wieder vom Schullehrer Leopold Franz Czurda. Das Inventar ${ }^{20}$ beschäftigt sich ausschließlich mit den auf dem Chor sich befindlichen Sachen - d. h. es ist kein Bestandteil eines umfangreicheren Inventars der Kirche. ${ }^{21}$ Die Übernahme des Dokumentes ist vom Lokalkaplan Caspar Thomas Tománek signiert. Diese Beschreibung befasst sich mit den Musikinstrumenten und Musikalien, die leider nicht mit den Namen der Komponisten verseht wurden. Doch Czurda hat immer den Gesamtanzahl angegeben, dann die brauchbare und die unbrauchbare Stücke. Dies ist für die Vorstellung der eigentlichen Kirchenmusikalischen

18 Diplomatische Transkription des Inventars - Anhang Nr. 2.

19 Es kommen anonyme Komponisten vor, sowie Namen mit, die mit einem Fragenzeichen versehen sind.

20 Diplomatische Transkription des Inventars - Anhang Nr. 3.

21 Unter den Akten befindet sich auch ein Inventar aus den Jahren 1817/1819. 
Betrieb sehr wichtig. Zu diesem Inventar ist kein Begleitsschreiben überliefert, aber vermutlich kann es wegen der neuen Besetzung der Lokalkapellanstelle entstanden sein.

\section{Musikalien bei Hrabiner Kirche}

Anfänglich wird die Aufmerksamkeit an die Musikalien im Besitz der Marianischen Kirche gerichtet, denn die Musikalieninventare immerhin seltener als die Musikinstrumenteninventare überliefert sind. Die Hrabiner Inventaren sind höchstwahrscheinlich nicht zwecks der Chordokumentation entstanden worden, sondern nur als ein Verzeichnis zuerst für das erzbischöfliche Konsistorium und weiter für das Pfarramt. Anhand dieser Angaben lassen sich einige Tatsachen über die Kirchenmusik in Hrabyně feststellen. Nachvollziehbar wird die Menge der Musikalien im Verlauf der ersten Hälfte des 19. Jahrhunderts - im Jahre 1804 befanden sich auf dem Chor insgesamt 156 Kompositionen, wovon die Mehrheit kleinere Musikstücke wie Arien, Motetten oder Offertorien bildeten. Gleich danach kommen die Messekompositionen, die ein Drittel des Repertoires darstellten. Beachtenswert sind auch die 20 Litaneien, deren Menge dem Wallfahrtsort zusammenhängt, obwohl es nicht eindeutig eingegeben ist, dass es sich um Marianische Litaneien handelt. Eine weitere Fundation stiftete den Gesang von Salve Regina jeden Samstagabend. Es muss noch die Tatsache erwähnt werden, dass das Inventar 1804 zweifellos Musikalien aus der zweiten Hälfte des 18. Jahrhunderts einschließt. Das bestätigen u. a. die Einträge im Inventar 1819 .

Als gewisser Zwischenteil kommt der Brief 1818, in dem mehr als die Hälfte der Musikalien aus dem vorherigen Inventar fehlt. Einige Gattungen verschwanden ganz, bei vielen wurde der Anzahl deutlich reduziert, doch im Gegenteil dazu kommt eine neue Gattung dazu - Sinfonien. ${ }^{22}$ Obwohl der Zustand allgemein als nicht ausreichend für Gestaltung guter Kirchenmusik bewertet wurde, sind die Sinfonien nicht ausdrücklich kommentiert. Es lässt sich vermuten, dass mindestens die Sinfonien während der Jahre 1804 und 1818 erworben werden mussten, demnach müssten sie relativ aktuelle Musikstücke präsentieren. Ein Jahr später sind (im Inventar 1819) insgesamt 94 Musikalien eingetragen. Das Begleitschreiben verlangt die Beschaffung der Kirchenmusikinstrumente, aber was die Musikalien betrifft, wird nur die ordentliche Übergabe gefordert. Das heißt, dass mindestens 22 neue Musikstücke gekauft wurden, darunter überwiegend kleinere Kompositionen wie Te Deum, Stationes zur Prozession zu Fronleichnam Christi oder Salve Regina.

Das Inventar 1819 ist das einzige, das die Namen der Komponisten erhält, aufgrund dessen sollen einige wichtige Anhaltspunkte angesprochen werden. Im Inventar stehen Nahmen der 52 Komponisten, die sich in zwei Gruppen teilen lassen - nicht identifizierbare und identifizierbare Komponisten. Zu der ersten Gruppe gehören nicht nur An-

22 Sinfonien befinden sich unter anderem im Inventar aus Kostelní Vydří. Mehr Informationen dazu bietet: KOUKAL, Petr. Symphonies in the thematic catalogue of Kostelní Vydří. In LOOS, Helmut (ed.). Musikgeschichte zwischen Ost- und Westeuropa: Symphonik - Musiksammlungen. Tagungsbericht Chemnitz 1995. Sankt Augustin, 1997. 
onyme, sondern auch Komponisten, ${ }^{23}$ deren Namen bereits im Inventar mit einem Fragezeichen versehen sind wie Daneck? Dazu kommen diejenigen, die nicht mehr bekannt sind und deren Werke nicht bis heute überliefert worden sind. Einige Komponisten sind zwar bekannt, allerdings nur anhand der Nachname kann nicht eindeutig entschieden werden, um welchen der Komponisten sich handeln könnte, weil die Nachnamen so geläufig sind, dass die Zuordnung äußerst fragwürdig wäre. Für alle können Müller, Miller oder Reiter genannt werden. In der Auflistung gibt es eine Menge der Schwankungen in der Orthographie bei ähnlichen Namen. Dabei kann vorausgesetzt werden, dass es in manchen Fällen um einen und denselben Komponisten gehen könnte - als Beispiel kann Miny, Minis, Minio, Mynis dienen. Darüber hinaus sind diese Diskrepanzen ein Zeichen dafür, dass der Verfasser - in diesem Falle der Regenschori Czurda - höchstwahrscheinlich diese Komponisten nicht mehr kannte, und dass diese Kompositionen älter sein müssen. Zu den unbekannten Komponisten gehören Baunzany, Daniel, Deisler, ${ }^{24}$ Galasch, Geisler, Keiser, Kunschner, Legimio, Ludislaw, Madlseder, Minis, Miny, Müller, Musial, Mynio, Mynis, Nahse, Raiter, Reiter, Regimie, Šchrosch und Wahner. Im Gegenteil dazu war es möglich achtundzwanzig Komponisten anhand des Namens, der angegebenen Gattung und der Tonart zu identifizieren. Dennoch auch hier sind gewisse Unklarheiten aufgetreten, weil einige Namen scheinen nur verwechselt zu sein - wie beispielsweise der Nachname Czurda und Gurda, doch eben in diesem Falle ist solch ein Fehler nicht zu vermuten, denn man annehmen kann, das der Verfasser Czurda darauf geachtet hatte.

Die Komponisten, die identifizierbar ${ }^{25}$ sind, können aus verschiedenen Aspekten betrachtet werden. Sie können aus einer chronologischen Sicht und deren Zugehörigkeit zu einzelnen Epochen in der Musikgeschichte eingeordnet werden. Die ältesten Musikalien stammen von Komponisten, deren musikalisches Schaffen in der ersten Hälfte des 18. Jahrhunderts liegt - wie Leonard Leo oder der Regenschori in Prager St.-VeitDom Johann Christoph Gayer. Sie stellen Ausnahmen dar und sowie die Musikstücke von Komponisten aus der zweiten Hälfte des 18. Jahrhunderts spiegeln eher als Relikte des früheren Musikgeschehens wider. Dazu kommen die Komponisten wie Mozart, ${ }^{26}$ Haydn, ${ }^{27}$ Zechner oder Ditters und Wanhal, Theny oder Wesely, die um die Jahrhundertwende lebte und tätig waren. Diese Ansicht in das Repertoire zeigt die Bemühungen um eine aktuelle Musik bei dem Kirchenmusikbetrieb zu spielen.

23 Es ist jedoch nicht auszuschließen, dass diese unbekannten Komponisten noch in der Zukunft identifiziert werden können, hauptsächlich dank der Digitalisierung der Musiksammlungen.

24 Einziges Musikstück, ein Divertimento, das mit dem Name Dreisler versehen wurde, befindet sich in einer Kollektion der Klaviermusik in der National Bibliothek der Tschechischen Republik (CZ-Pu).

25 Für die Identifikation wurden die üblichen aktuellen Handbücher und Übersichtswerke, wie z.B. Musik in Geschichte und Gegenwart, Grove, SČHK, usw. und beziehen sich auf heutigen Forschungsstand.

26 Die Autorschaft von Mozart muss mit Zweifel betrachtet werden. Es gibt in mährischen Quellen eine Menge der Kontrafakturen, aber auch von falschen Zuschreibungen, die oft Mozart betreffen. Vgl. SEHNAL, Jiří. Quellen zu Mozarts Kirchenwerken in Mähren. In Mozart-Jahrbuch 1986, Bärenreiter, Kassel, 1986, S. 31.

27 Bei Haydn ist es nicht möglich zu entscheiden, ob es sich um Joseph oder Michael Haydn handelt. Vermutet wird jedoch, dass es wahrscheinlich um Michael Haydn geht, weil seine Kompositionen in den Ländern der böhmischen Krone verbreitet wurden. Vgl. SEHNAL, Jiří. Mše Josefa Haydna na Moravě v 18. a 19. století. In Joseph Haydn a hudba jeho doby. Bratislava 1984, s. 115-119. 
Das Inventar belegt unter anderem den lokalen Musikbetrieb nicht nur in Hrabin, sondern auch in Schlesien allgemein, denn zehn der Komponisten hatten eine direkte Beziehung zur Schlesien, bzw. zu den Kapellen der Adeligen in Schlesien. Zur Kapelle vom Graf Chorinsky gehörten Puschmann, Silvester oder Gurda, die sich mit dem Grafen im Opava (Troppau) unterließen, aber auch im Schloss in Velké Hoštice (Groß Hoschütz), in dem auch Theateraufführungen stattfanden. Eine bedeutende Persönlichkeit war Carl Ditters von Dittersdorf, der als Kapellmeister des Fürstbischofs Philipp Gotthard von Schaffgotsch in Javorník (Jauernik) auf dem Schloss Jánský vrch (Johannesberg) tätig war.

Andere ideelle Gruppe der Komponisten bilden die Schullehrer und gleichzeitig Regenschoris, die bei ihrer Tätigkeit komponierten. ${ }^{28}$ Aus den Hrabiner Aktenmaterial geht allerdings nicht hervor, dass es eine Pflicht sein sollte, als Hrabiner Schullehrer auch komponieren zu müssen. Trotzdem wurden drei hiesige Kantoren Köhler, Czurda und Opavsky ${ }^{29}$ ins Inventar eingetragen. $\mathrm{Zu}$ den Komponisten unter Schullehrern ist Gurda zuzuordnen, denn er war zu derselben Zeit als Musiker bei Grafen Chorinsky und Regenschori in Velké Hoštice angestellt. Vor allem als Regenschori arbeitete Schnitzler, dessen Wirkungsort die Kirche und Schule in Rýmařov (Römerstadt) war. Die Annahme liegt nah, dass es unter den nicht identifizierbaren Komponisten einige aus dem Hrabiner, resp. Schlesischen Umfeld befinden können.

Überraschenderweise blieb die Gesamtzahl der Musikalien gleich wie im Inventar 1819. Doch die einzelnen Eintragungen unterscheiden sich voneinander. Die Angaben darüber, ob die Musikalien brauchbar oder unbrauchbar sind, bieten die Möglichkeit, bessere Übersicht zu bekommen, wie die Chorausstattung tatsächlich aussehen könnte. Von der Gesamtsumme können nach Czurdas Meinung etwa mehr als ein Drittel der Musikalien für anständige Kirchenmusik benutzt werden. Beispielsweise ist aus den acht Sinfonien, die im Inventar 1819 notiert sind, nur die Hälfte verwendbar. Diese Zahlen weisen auf zwei Möglichkeiten hin: entweder wurden die Musikalien beschädigt oder zerstört, oder sie entsprachen nicht mehr dem aktuellen Musikstil. Dieser Grund kann wohl vermutet werden, denn die Regenschoris sind in den Instruktionen oft dazu ermahnt, neue und zeitgenössische Musik auf dem Chor zu spielen. Deshalb wird angenommen, dass die Musikstücke von Komponisten wie Gayer, Leo, Galuppi oder Brixi am Anfang des 19. Jahrhunderts nicht mehr gespielt wurden und als unbrauchbar betrachtet sein könnten. Gewiss lässt sich behaupten, dass das Te Deum von einem Anonymus und Stationes zur Prozession zu Fronleichnam Christi von Gurda nicht mehr brauchbar waren. Im Gegenteil dazu wurden Vidi Aquam von Gurda als brauchbar bezeichnet. Neulich befanden sich auf dem Chor drei neue Vespern, die im Inventar 1819 fehlen.

28 Sehnal äußert sich, dass es oft nicht über Pflichtkompositionen ging, sondern eher um eigenen Ehrgeiz oder aber um eine örtliche Profilierung der Kirche oder Lokalität gehen könnte. Vgl. SEHNAL, Jiř́í. Figurální hudba ve farních kostelích na Moravě v 17. a 18. století. In: Hudebni věda, roč. 23 Praha: Academia, 1996., S. 172. Nicht zuletzt wusste der Regenschori über Fähigkeiten und Besetzung des Chores und konnte den Bedingungen die Kompositionen anpassen.

29 Bei Opavsky kann sich auch nur um eine zufällige Namensgleichheit handeln. 
Ein relativ selbständiges Kapitel bilden die Gesangbücher, oder Cancionalia. Sie sind am meisten bei den Musikinstrumenten eingetragen - das ist der Fall nicht nur in Hrabyně. Diese Praxis war im 18. Jahrhundert auch in anderen Inventaren aus Mähren und Böhmen geläufig. Deshalb sind Gesangbücher nur im Inventar 1804, Brief 1818 und Inventar 1832 zu finden und ihre Anzahl sinkt sowie die Zahlen bei den Musikalien und Musikinstrumenten. In Hrabyně standen im Jahre 1804 vier Exemplare des Thomas Frycaj Gesangbuches zur Verfügung, dabei könnte es sich entweder um die erste Ausgabe aus dem Jahr 1788 mit dem Titel Písně duchovni podle kancionálu německého handeln, oder um die zweite Ausgabe mit dem Titel Ouplná kníha duchovních pisní katolických aus dem Jahre 1801. ${ }^{30}$ Später sind nur zwei Exemplare von diesem Gesangbuch auf dem Chor notiert, die noch im Jahre 1832 als verwendbare bewertet wurden. Daneben wurde im Kirchenvermögen noch „ein böhmisches Cancional“, das zuerst in den Akten im Jahre $1804 \mathrm{zu}$ finden ist, und noch im Jahre 1832 benutzbar sei.

\section{Musikinstrumente im Besitz des Wallfahrtsortes}

In der Abhandlung Figurálni hudba ve farních kostelich na Moravě v 17. a 18. století weist Jiří Sehnal auf den Zuwachs der Figuralmusik bei der Liturgie in der zweiten Hälfte des 18. Jahrhunderts hin, trotz zahlreicher Verbote von Blasinstrumente und Josephinischer Kirchenreform. Dies betrifft kleinere Lokalitäten wie Dorfkirchen und ländliche Wallfahrtsorte, weil die Musik zu den Repräsentationszwecken gleich wie künstlerische Verschönung der Kirche, prächtige Altare und verzierte Ornate gehörte. All das wurde in Hrabyně noch gesteigert, denn das Dorf gewann an seiner Bedeutung eben in der Hälfte des 18. Jahrhunderts. ${ }^{31}$ Dabei halfen nicht nur Privilegien wie die Märkte veranstalten zu können, sondern auch die regelmäßige Wallfahrte und eine große Menge an Pilger. Die Kirchenfeste gehörten zu damaligen „hohen Festen“, bei deren der Schullehrer verpflichtet war, Figuralmusik zu gewährleisten.

Wie schon oben bemerkt wurde, stammt die erste Erwähnung von der Figuralmusik aus dem Jahre 1761, in dem es eine Fundation festgelegt wurde. Diese Fundation sollte sichern, dass der Schullehrer und zwei Vokalisten die Liturgie mit dem Choral und mit der Instrumentalmusik begleichen werden. Den allerersten Beleg bildet jedoch der Einschrift im Archiv, dass die Figuralmusik am Sonntag am 24. November 1777. Das heißt, dass es vom 1761 bis 1804 mindestens 26 Musikinstrumente besorgt wurden, darunter sind eine Orgel mit 12 Registern und ein Positiv mit 6 Registern zugezählt. Hauptsächlich waren im Vermögen der Kirche die Blas- und Streichinstrumente, sowie das Zubehör dazu. Bis auf eine Ausnahme ist der Zustand der Instrumente nicht reflektiert, deshalb kann vorausgesetzt werden, dass die Instrumente brauchbar waren. Die einzige Ausnahme sind zwei alte Claviere, die im Unterricht verwendet wurden.

30 Vgl. SEHNAL, Jiří - VYSLOUŽIL, Jiří. Dějiny hudby na Moravě. Brno: Muzejní a vlastivědná společnost, 2001, S. 148.

31 Vgl. MALURA, Jan. Hora Olivetská Matěje Tannera a kultura poutních míst v baroku. In TANNER, Matěj. Hora olivetská. Brno: Host, 2001, S. 9. 
In folgenden Dokumenten sinkt rapid die Anzahl der Instrumente - im Brief 1818 sind auf dem Chor nur acht Musikinstrumente geblieben. ${ }^{32}$ Laut dieses Briefes war der Violon ruiniert und im gleichen Zustand befanden sich die Waldhörner, Trompeten und Viola. Diese Veränderung könnte von der Zeit bedingt sein, weil sie nach 14 Jahren beschädigt sein dürften. Möglich ist ebenfalls, dass nach der Besetzung der Schullehrersund Regenschoristelle einige von den Instrumentalisten entlassen werden könnten und deshalb eigene Instrumente mitnehmen könnten. Dafür spricht jedoch, dass im 18. Jahrhundert die Musikinstrumente zwar üblicherweise zur festen Ausstattung der Kirchen gehörten, jedoch später wurden sie immer von dem Preis zugänglicher, und deshalb waren z. B. die Streichinstrumente wie Geigen oft im Besitz einzelnen Musiker. Was die Kircheninstrumente betrifft, ist zu ihrer Zustandsverschlimmerung vermutlich während der Tätigkeit von Schullehrer Köhler und Jerzabek gekommen. In dem Aktenmaterial sind Beschwerden über das Benehmen beiden Lehrer erhalten. Im Visitationsprotokoll über Tätigkeit von Köhler aus dem Jahre 1814 steht, dass Köhler „die Musik Instrumenta in den schlechtesten Stand [hat]. "33 Sein Nachfolger Jerzabek sollte eine Lösung für diese unglückliche Situation sein, jedoch wie aus den weiteren Protokollen und anderen Akten hervorgeht, verbesserte sich dieser Zustand nicht, es habe sich eher verschlechtert. Ein Jahr später kommt es zu weiteren Beschwerden, denn Jerzabek nicht nur keine Musikinstrumente pflegt, sondern auch keine Vokalisten lehret, wofür er doch bezahlt wird. Es wurden auch seine Schulmethoden, seine Trunkheit, Unkeuschheit und Unsittlichkeit sowie wegen den Scherzen über Kirche und Religion kritisiert.

Nächsten Übersicht der Kirchenmusikinstrumente stammt erst aus dem Jahre 1832. In dieser Zeit befanden sich auf dem Chor nur elf Musikinstrumente, dabei gab es weder Kirchengeigen, noch Viola, sondern nur Bassetl, drei Trompeten, Timpani und zwei Waldhörner - neu ist hier nur eine Klarinette. Im Gegenteil zum Brief werden die Musikinstrumente als brauchbare bezeichnet, deshalb lässt die annehmen, dass einige davon nach der Ermahnung im Jahre 1819 neu besorgt wurden. Obwohl es scheinbar in der Kirche an Geigen mangelt, bedeutet die Absenz der Geigen natürlich nicht, dass sie nicht mehr gebraucht wurden. Am Anfang des 19. Jahrhunderts wurden sie ein relativ zugänglicher Artikel, demnach könnten sie im Besitz der Musiker sein. ${ }^{34}$ Dazu kommt, dass Czurda in seiner Beschwerde schrieb, er müsse eigene Musikinstrumente verwenden. Von daher ist es schon möglich, dass er weiter seine eigenen Instrumente neben den Kircheninstrumenten benutzte.

Um eine bessere Vorstellung zu erhalten, ist es nötig, die Daten aus Hrabyně mit anderen Kirchen in der Nähe zu vergleichen. Zu ähnlich großen Lokalitäten im Schlesien gehörte beispielsweise Strumień ${ }^{35}$ (Schwarzwasser) in Teschener Schlesien. Hier ist ebenso im Jahre 1804 ein Inventar der Musikinstrumente entstanden - auf dem Chor befanden sich damals Musikinstrumente, darunter sind sechs Instrumente als alt,

32 Darunter ist anscheinend nicht die Orgel, bzw. auch Positiv nicht gezählt.

33 DOZA WIEN, Fond Mei, Inv. Nr. 432/1.

34 Vgl. SEHNAL, Jiří. Obsazení v chrámové hudbě 17. a 18. století. Hudebni věda 8. S. 243.

35 Polsky Strumień, německy Schwarzwasser, se dnes nachází v polské části Těšínska. 
abgebraucht oder schlecht beschrieben. ${ }^{36}$ Indra führt eine These ein, dass die Kirchen in Teschener Schlesien besser als die Kirchen in Troppauer Teil des Schlesiens ausgestattet wurden. Beim Vergleich der 26 Hrabiner Kircheninstrumente und bloßen 15 aus Strumień, könnte diese These widerlegt werden. Um dies aber sicher sagen zu können, müssten die Zustände mehreren Kirchen in beiden Gebieten genauer untersucht werden. Überdies muss in Betracht gezogen werden, dass es im Falle der Hrabiner Kirche um einen Wallfahrtsort geht, der vermutlich bessere Geldquellen hatte.

Wertvoll sind in diesem Zusammenhang einige Berichte aus dem Anfang des 19. Jahrhunderts vom Kantor Mathias Johann Schenk, der in Bělotín (Bölten) bei Hranice na Moravě (Mährisch Weißkirchen) seinen Beruf als Lehrer und Regenschori ausübte. ${ }^{37} \mathrm{Im}$ Jahre 1809 verfasste er seine Erinnerungen, die er später im Jahre 1822 ergänzte. Er berichtet darüber, dass den Wünschen nach er sich entschied, eine Kirchenmusik mit den Kindern einzuüben. Dafür brauchte er nur eine erste Geige, drei Stück von zweiten Geigen und eine Klarinette, die der Schulhelfer spielte. Die erste Produktion fand dann am 15. August 1812 statt. Erst danach seien die bestellten Trompeten aus Olomouc (Olmütz) für 30 fl. worden, mit denen sie den Introitus und Intraden spielten, was eine noch gröBere Freude bei den Bewohnern verursachte. Am 30. Mai 1813 bekam der Schullehrer zu den Trompeten noch Timpani im Wert von 80 fl., die ebenso in Olmütz gekauft wurden. ${ }^{38}$ Diese subjektiven Eindrücke des Schullehrers widerspiegeln die Wahrnehmung der Kirchenmusik und ihre Wichtigkeit und Bedeutung für damalige Dorfeinwohner. Auf den ersten Blick ist es klar, dass aus unseren Sicht relativ kleine Besetzung (acht Musikinstrumente) reichte, um den Zuhörer die verlangte Figuralmusik zu sichern. Im Gegenteil dazu war es fünf Jahre später für den Hrabiner Schullehrer nicht genügend, wenn er acht ältere Musikinstrumente zur Verfügung hatte.

\section{Zustand der Hrabiner Orgel}

In den Inventaren gibt es auch wichtige Informationen über Orgel. Denn es ist dank der Visitationsprotokolle bekannt, dass es in Hrabyně zwischen den Jahren 1671 und 1673 ein Regal gab. ${ }^{39}$ Von Jiř́i Sehnal wurde dann das Bau einer neuen Orgel mit zwölf Registern und einem Manual in das Jahr 1757 festgestellt. ${ }^{40}$ Die erste Erwähnung der Orgel in den untersuchten Aktenmaterialien befindet sich im Protokoll aus dem Jahre

36 Vgl. INDRA, Bohumír. Archivní materiály k starším hudebním dějinám Slezska. In Slezský sbornik 56/1958, S. 122 .

37 Mathias Schenk wurde die Aufmerksamkeit in folgender Abhandlung gewidmet: MAŇAS, Vladimír. Kantor - Několik příkladů místo syntézy. In Člověk na Moravě ve druhé polovině 18. století. Brno: CDK, $2008 . \mathrm{S}$. 95-104.

38 Moravský zemský archiv v Brně, G 10 Sbírka rukopisů, sign. 1160. Für den Hinweis auf diesen Material bin ich Mgr. Vladimír Maňas, Ph.D., sehr dankbar.

39 In den späteren Visitationsprotokollen, die aus den Jahren 1686 bis 1691 stammen, ist es nicht mehr eingetragen. Vgl. SEHNAL, Jiří. Barokni varhanářstvi na Moravě. Brno: Muzejní a vlastivědná společnost, 2003. S. 144

40 Ibidem., S. 24 
1791, in dem die Pflichte des Schullehrers aufgelistet sind. Im Inventar 1804 hatte die Orgel laut der Angaben ebenfalls zwölf Register, jedoch zwei Manuale und einen Positiv mit sechs Registern.

Weitere ausführlichere Auskunft bietet ein Entwurf über geplante Gesamtreparatur der Orgel, die von Johann Kuttler ${ }^{41}$ im Jahre 1819 geschrieben wurden. Aus seinem Vorschlag ergibt sich, dass die Orgel nicht mehr in einem entsprechenden Zustand waren, um den Musikbetrieb zu gewährleisten. Kuttler schrieb, dass es die Ventile gewechselt werden sollten, weiter auch beide Manuale neugestaltet werden sollen. Getauscht werden sollte seiner Ansicht nach unter anderen der Blasebalg, sowie alle hölzernen Pfeifen. Am Ende des Entwurfes steht noch, dass ein Register völlig abgerissen sein solle und deshalb durch einen neuen ersetzt sein solle. Als einen notwendigen Bestandteil der Reparatur gibt er die Einstimmung der Orgel an. Ob die Reparatur zustande kam, und ob sie von Kuttler tatsächlich durchgeführt wurde, kann nicht anhand der Akten aus dem DOZA eindeutig bestätigt werden. An dieser Stelle hilft Wolnys Publikation Kirchliche Topographie von Mähren, meist nach Urkunden und Handschriften, in der angegeben ist, dass die Orgel im Jahre 1823 repariert und erneut wurde. ${ }^{42}$ Dies stimmt dann mit dem Inventar 1832 überein, denn hier Czurda schreibt, dass die Orgel nicht mehr zwölf, sondern dreizehn Register haben. Die Reparatur ist zwar nicht erwähnt, allerdings ist ihr Zustand als brauchbar bewertet. Darum wird davon ausgegangen, dass Kuttler sich in dieser Zeitspanne der Reparatur der Hrabiner Orgel gewidmete.

\section{Fazit}

Schlesischer Wallfahrtsort Hrabyně gewann an seiner Bedeutung in der zweiten Hälfte des 18. Jahrhunderts. Hand im Hand damit geht der Aufstieg des Musikbetriebes, dass in vorgelegter Abhandlung geschildert wurde. Die erhaltenen Inventare und Aktenmaterial vermitteln einen Einblick in die Musik in Troppauer Land, obwohl es keine Musikalien oder Kircheninstrumente erhalten haben. Somit wurden die bloße zwecks kirchlicher Dokumentation verfassten Auflistungen zu einer primären Quelle, die nicht nur für lokale musikgeschichtliche Topographie von Bedeutung ist, sondern auch die Erkenntnisse über den Musikbetrieb und seinen langsamen Verfall zu finden ermöglicht.

Studie pripravena v rámci projektu Grantové agentury České republiky Hudební inventáře raného novověku v českých zemích (GA16-17615S).

41 Über Kuttner schreibt ausführlicher Jiří Krátký in der Studie: KRÁTKÝ, Jiří. Johann Kuttler - varhanář slezského pohraničí. In: Časopis Slezského zemského muzea. B, Vědy historické. Opava: Slezské zemské muzeum, 2015.

42 WOLNY, Gregor. Kirchliche Topographie von Mähren, meist nach Urkunden und Handschriften. Brünn: Selbstverlag, 1863. S. 308. 


\section{Bibliography}

\section{Sources and literature}

Deutschordens Zentralarchiv Wien, Fond Mei (Meisterthum) Inv. Nr. 432/1.

Deutschordens Zentralarchiv Wien, Fond Mei (Meisterthum) Inv. Nr. 432/2.

Deutschordens Zentralarchiv Wien, Fond Mei (Meisterthum) Inv. Nr. 433/1.

Moravský zemský archiv v Brně, G 10 Sbírka rukopisů, Sign. 1160.

EICHLER, Karel. Poutni mista a milostivé obrazy na Moravě a v rakouském Slezsku. Brno: Dědictví sv. Cyrilla a Methoděje. Díl první, část druhá, 1888.

HOSÁK, Ladislav. Historický místopis země Morauskoslezské. VIII. Opauský kraj. Praha: Společnost přátel starožitností československých, 1937.

KOUKAL, Petr. Symphonies in the thematic catalogue of Kostelní Vydří. In LOOS, Helmut (ed.) Musikgeschichte zwischen Ost- und Westeuropa: Symphonik - Musiksammlungen. Tagungsbericht Chemnitz 1995. Sankt Augustin, 1997.

KRAMÁŘOVÁ, Helena. Chrámové inventáre hudebnin z prèlomu 18. a 19. století. Hrabyňské inventáre [online]. Brno, 2013 [cit. 2016-09-28]. Bakalářská práce. Masarykova univerzita, Filozofická fakulta. Vedoucí práce Vladimír Maňas Zugänglich unter: 〈http://is.muni.cz/th/383033/ff_b/>.

KRÁTKÝ, Jiří. Johann Kuttler - varhanář slezského pohraničí. Časopis Slezského zemského muzea. B, Vědy historické. Opava: Slezské zemské muzeum, 2015.

MALURA, Jan. Hora Olivetská Matěje Tannera a kultura poutních míst v baroku. In TANNER, Matěj. Hora olivetská. Brno: Host, 2001.

MAŇAS, Vladimír - ORLITA, Zdeněk - POTƯČKOVÁ, Martina. Zbožných duši úl. Náboženská bratrstva v kultuře raněnovověké Moravy. Olomouc: Muzeum umění Olomouc, 2010.

MAŇAS, Vladimír. Hudba v Hlučíně v období raného novověku. Opus musicum, Brno, roč. 34, č. 5, 2002. s. 4-12.

MAŇAS, Vladimír. Kantor - Několik příkladů místo syntézy. In Člověk na Moravě ve druhé polovině 18. století. Brno: CDK, 2008. S. 95-104.

MAŇAS, Vladimír. Náboženská bratrstva olomoucké (arci)diecéze do josefínských reforem [online]. Brno, 2003 [cit. 2016-09-30]. Diplomová práce. Masarykova univerzita, Filozofická fakulta. Vedoucí práce Bronislav Chocholáč Dostupné z: 〈http://is.muni.cz/th/13678/ff_m/〉.

SAMEK, Bohumil. Umělecké památky Moravy a Slezska. 1., A-I. Praha: Academia, 1994.

SEHNAL, Jiří - VYSLOUŽIL, Jiří. Dějiny hudby na Moravě. Brno: Muzejní a vlastivědná společnost, 2001.

SEHNAL, Jiří. Barokni varhanářstvi na Moravě. Brno: Muzejní a vlastivědná společnost, 2003.

SEHNAL, Jiří. Figurální hudba ve farních kostelích na Moravě v 17. a 18. století. Hudebni věda, roč. 23 Praha: Academia, 1996.

SEHNAL, Jiří. Mše Josefa Haydna na Moravě v 18. a 19. století. In Joseph Haydn a hudba jeho doby. Bratislava 1984, s. 115-119.

SEHNAL, Jiří. Obsazení v chrámové hudbě 17. a 18. století. Hudebni věda 8. S. 236-247.

TROJAN, Jan. Kantoři na Moravě a ve Slezsku v 17.-19. století: jejich sociálni postaveni, společenská funkce a význam ve vývoji národni hudebni kultury. Brno: Muzejní a vlastivědná společnost, 2000.

WOLNY, Gregor. Kirchliche Topographie von Mähren, meist nach Urkunden und Handschriften. Brünn: Selbstverlag, 1863. 


\section{Anhang Nr. 1 Inventar 1804}

\begin{tabular}{|l|c|}
\hline Ob dem Chor ist befindlich: & Stück \\
\hline ein Orgel mit 12 Mutationen und dopeler Claviatur & 1 \\
\hline ein Positiv mit 6 Mutationen & 1 \\
\hline alte Clavier zum Schulunterricht & 2 \\
\hline Pauken samt Schlägeln & 2 \\
\hline Trompeten & 5 \\
\hline Waldhörner & 4 \\
\hline Mundstück zu Trompeten & 7 \\
\hline Krumbbögen und Stockerl zum Trompeten & 4 \\
\hline Violon samt Schlüsl und Bogen & 1 \\
\hline Bassetl & 1 \\
\hline Geigen und 1 St Bratschen Simul & 6 \\
\hline Ein geschriebenes Choral Rituale & 1 \\
\hline Thomas Fričagische Gesangbücher & 4 \\
\hline Ein böhmisches Cantional & 1 \\
\hline
\end{tabular}

\begin{tabular}{|l|c|}
\hline Musicalien & Kusů \\
\hline Messen lange, und kurze, samt Pastor: Eben & 47 \\
\hline Lytaneyen & 20 \\
\hline Requiem Messen & 9 \\
\hline Vespern & 2 \\
\hline Arien, oder Moteta, ein und mehr stimmig & 54 \\
\hline Pangelingua & 9 \\
\hline Te Deum & 1 \\
\hline Stabat Mater & 1 \\
\hline Stationes pro Festo Teophoriae & 3 \\
\hline Salve Regina & 3 \\
\hline Regina ceoli & 5 \\
\hline Vidi aquam & 1 \\
\hline Rorate & 1 \\
\hline
\end{tabular}




\section{Anhang Nr. 2 Inventar 1819}

\begin{tabular}{|c|c|c|c|}
\hline Numer & Missa & Thon & Author \\
\hline \multicolumn{4}{|l|}{ Missa } \\
\hline 1. & ett. & $A$ & Clement \\
\hline 2. & ett. & $\mathrm{D}$ & Raiter \\
\hline 3. & ett. & $\mathrm{A}$ & Geisler \\
\hline 4. & ett. & Dis & Ett \\
\hline 5. & ett. & $G$ & Aumann \\
\hline 6. & ett. & $\mathrm{B}$ & ett \\
\hline 7. & ett. & $D$ & Geisler \\
\hline 8. & ett. & $\mathrm{A}$ & Brixy \\
\hline 9. & ett. & $\mathrm{D}$ & ett \\
\hline 10. & ett. & $\mathrm{D}$ & ett \\
\hline 11. & ett. & $\mathrm{D}$ & Hann \\
\hline 12. & ett. & $\mathrm{A}$ & Opavsky \\
\hline 13. & ett. & $C$ & Hann \\
\hline 14. & ett. & $\mathrm{D}$ & ett \\
\hline 15. & ett. & $C$ & Šimon \\
\hline 16. & ett. & $\mathrm{D}$ & Wahner \\
\hline 17. & ett. & $\mathrm{C}$ & Reiter \\
\hline 18. & ett. & $\mathrm{C}$ & Clement \\
\hline 19. & ett. & $\mathrm{B}$ & Heydn \\
\hline 20. & ett. & $C$ & Bryxy \\
\hline 21. & ett. & $C$ & Puschmann \\
\hline 22. & ett. & $C$ & ett \\
\hline 23. & ett. & $C$ & Müller \\
\hline 24. & ett. & $D$ & Schnitzler \\
\hline 25. & ett. & $\mathrm{C}$ & ett \\
\hline 26. & ett. & $C$ & Vogel \\
\hline 27. & ett. & C & ett \\
\hline \multicolumn{2}{|l|}{ Summa } & 27 & \\
\hline
\end{tabular}

\begin{tabular}{|c|c|c|c|}
\hline Numer & Simpho. & Thon & Author \\
\hline \multicolumn{4}{|c|}{ Sinphonien } \\
\hline 1. & ett. & $D$ & Šchrosch \\
\hline 2. & ett. & G & Vešely \\
\hline 3. & ett. & C & Vanhal \\
\hline 4. & ett. & G & Unbekannt \\
\hline 5. & ett. & C & Vanhal \\
\hline 6. & ett. & A & Ditters \\
\hline 7. & ett. & G & Ett \\
\hline 8. & ett. & D & Lochelio \\
\hline \multicolumn{2}{|l|}{ Summa } & 8 & \\
\hline \multicolumn{4}{|c|}{ Offertorium } \\
\hline 1. & ett. & $\mathrm{C}$ & Bryxy \\
\hline 2. & ett. & $\mathrm{C}$ & \begin{tabular}{|l|} 
Mynio \\
\end{tabular} \\
\hline 3. & ett. & C & Baunzany \\
\hline 4. & ett. & $\mathrm{G}$ & Mynis \\
\hline 5. & ett. & G & Navratil \\
\hline 6. & ett. & $\mathrm{D}$ & Galasch \\
\hline 7. & ett. & C & Brixy \\
\hline 8. & ett. & C & Ett \\
\hline 9. & ett. & $\mathrm{D}$ & Köhler \\
\hline 10. & ett. & C & Minis \\
\hline 11. & ett. & $C$ & Gurda \\
\hline \multicolumn{2}{|l|}{ Summa } & 11 & \\
\hline \multicolumn{4}{|l|}{ Moteto } \\
\hline 1. & ett. & $\mathrm{F}$ & Madlseder \\
\hline 2. & ett. & $\mathrm{A}$ & Gurda \\
\hline 3. & ett. & $\mathrm{G}$ & \begin{tabular}{|l|} 
Lochelio \\
\end{tabular} \\
\hline 4. & ett. & $\mathrm{A}$ & Ett \\
\hline
\end{tabular}


Die Figuralmusik in Hrabyně um die Wende des 19. Jahrhunderts - Hrabiner Inventare

\begin{tabular}{|c|c|c|c|c|c|c|c|}
\hline Numer & Moteto & Thon & Author & Numer & & Thon & Author \\
\hline \multicolumn{4}{|l|}{ Aria } & \multicolumn{4}{|c|}{ Tedeum laudamus } \\
\hline 1. & ett. & $\mathrm{F}$ & Gallupy & 1. & ett. & $\mathrm{D}$ & unbekannt \\
\hline 2. & ett. & $\mathrm{G}$ & Daniel & \multicolumn{4}{|c|}{ Vidi aqua } \\
\hline 3. & ett. & $\mathrm{F}$ & Daneck? & 1. & ett. & $\mathrm{D}$ & Gurda \\
\hline 4. & ett. & $\mathrm{F}$ & Zechner & \multicolumn{4}{|c|}{ Requiem } \\
\hline 5. & ett. & $E$ & Looš & 1. & ett. & $\mathrm{D}$ & ett \\
\hline 6. & ett. & $\mathrm{G}$ & Ludislaw & 2. & ett. & $\mathrm{Cb}$ & Regemie \\
\hline 7. & ett. & Dis & Brixia & 3. & ett. & $\mathrm{F}$ & Šedlačzek \\
\hline 8. & ett. & Dis & Brixio & 4. & ett. & $\mathrm{F}$ & \begin{tabular}{|l|} 
Legimio \\
\end{tabular} \\
\hline 9. & ett. & $\mathrm{A}$ & Leo & 5. & ett. & $\mathrm{B}$ & Galasch \\
\hline 10. & ett. & $\mathrm{F}$ & Nahse & 6. & ett. & $\mathrm{C}$ & Musial \\
\hline \multicolumn{4}{|c|}{ Pangelingua } & 7. & ett. & $\mathrm{Db}$ & Deisler \\
\hline 1. & ett. & $\mathrm{D}$ & Navratil & 8. & ett. & $\mathrm{C}$ & Miny \\
\hline 2. & ett. & $\mathrm{C}$ & Navratil & 9. & ett. & $\mathrm{F}$ & Looš \\
\hline 3. & ett. & $\mathrm{G}$ & Navratil & \multicolumn{4}{|l|}{ Litany } \\
\hline 4. & ett. & $\mathrm{G}$ & unbekannt & 1. & ett. & $\mathrm{G}$ & Holzbauer \\
\hline 5. & ett. & $C$ & Köhler & 2. & ett. & $C$ & Navratil \\
\hline 6. & ett. & $C$ & Czurda & 3. & ett. & $C$ & ett \\
\hline 7. & ett. & $C$ & Šilvester & 4. & ett. & $\mathrm{F}$ & Puschmann \\
\hline 8. & ett. & $D$ & Theny & 5. & ett. & $\mathrm{D}$ & Clement \\
\hline 9. & ett. & $C$ & unbekannt & 6. & ett. & $\mathrm{D}$ & ett \\
\hline 10. & ett. & $C$ & Czurda & 7. & ett. & D & Kohaut \\
\hline 11. & ett. & $C$ & Mozart & \multicolumn{2}{|l|}{ Summa } & \multicolumn{2}{|l|}{7} \\
\hline \multicolumn{4}{|c|}{ Asperiges } & & & & \\
\hline 1. & ett. & $C$ & Köhler & & & & \\
\hline 2. & ett. & $C$ & Kunschner & & & & \\
\hline \multicolumn{4}{|c|}{ Regina [coelli] } & & & & \\
\hline 1. & ett. & $\mathrm{B}$ & Keiser & & & & \\
\hline 2. & ett. & $\mathrm{D}$ & Gurda & & & & \\
\hline 3. & ett. & A & Geyer & & & & \\
\hline \multicolumn{4}{|c|}{ Stationes } & & & & \\
\hline 1. & ett. & $\mathrm{D}$ & Gurda & & & & \\
\hline
\end{tabular}




\section{Anhang Nr. 3 Inventar 1832}

ein Orgel mit 13 Mutationen

ein Positiv mit 6 Mutationen

Pauken samt Schlägeln

Trompeten

Waldhörner

Clarinete

Mundstück zu Trompeten

Mundstück zu Waldhörner

Krumbögen und Stockerl zum Trompeten

ett. .. ett zum Waldhörner

ett. .. ett k lesním horům

Violon samt Bogen

Bassetl

Geigen und Bratschen

Ein geschriebenes Rytual

Thomas Fricaisches Gesangbücher

Ein Böhmisches Cantional

\begin{tabular}{|l|c|c|c|}
\hline Musicalien & Stücke & Benutzbare & Unbenutzbare \\
\hline Messen & 28 & 8 & 20 \\
\hline Lytanien & 7 & 1 & 6 \\
\hline Requiem & 2 & 2 & - \\
\hline Vešper & 3 & 3 & - \\
\hline Arien, Moteta et Offertorien & 26 & 5 & 21 \\
\hline Symphonien & 8 & 4 & 4 \\
\hline Pangelingua & 11 & 6 & 5 \\
\hline Te Deum & 1 & - & 1 \\
\hline Stationis pro festo Teophoria & 1 & - & 1 \\
\hline Stabat Mater & - & - & - \\
\hline Salve Regina & 1 & 1 & - \\
\hline Regina cölli & 3 & 1 & 2 \\
\hline Vidi aquam & 1 & 1 & - \\
\hline Rorate & 1 & - & 1 \\
\hline Asperies & 1 & 1 & - \\
\hline Veni Sancte Spiritus & - & - & - \\
\hline Veni Creator Spiritus & - & - & \\
\hline
\end{tabular}


